\title{
Fetal heart rate variation with umbilical haematoma
}

\author{
S. Ballas*, S. Gitstein and J. Kharasch \\ Department of Obstetrics and Gynecology, Rebecca Sieff Government Hospital, Safed, Israel.
}

\begin{abstract}
Summary: Umbilical cord haematoma is an infrequent condition associated with high perinatal and fetal mortality and morbidity. This report describes a rare case of umbilical cord haematoma associated with loss of fetal beat to beat variation during labour. The infant exhibited mild asphyxia only.

Previous publications are reviewed and fetal heart rate changes associated with umbilical cord haematoma are discussed.
\end{abstract}

\section{Introduction}

Haematoma of the fetal umbilical cord is a rare condition occurring during late pregnancy and labour. Approximately 50 cases have been published to date. Although Hyrtl first described this finding in 1871, little is known about its aetiology, pathogenesis, fetal heart rate changes and, if any, intrapartum signs. No mention is made of fetal heart rate patterns. Whether or not there are clinically significant signs or symptoms of this condition remains an open question.

\section{Case report}

A 23 year old gravid 1, para 0 presented at 41 gestational weeks in active labour following a normal prenatal course. Admission urine and blood pressure were normal. The uterus was of appropriate gestational size with a fully effaced and dilated cervix of $4 \mathrm{~cm}$. Routine amniotomy revealed heavy meconium staining. An internal tococardiograph was utilized and labour lasted $5 \mathrm{~h}$. During labour the fetal heart tracing showed loss of beat to beat variation before as well as after the analgesia of mepiridine $50 \mathrm{mg}$ and promethazine $25 \mathrm{mg}$ (Figures 1 and 2). A normal female infant with Apgar score 6 was delivered spontaneously. The weight was $4150 \mathrm{~g}$. The fetus, covered with thick meconium, required suction of the upper airways and administration of oxygen and positive pressure ventilation. Apgar score at $5 \mathrm{~min}$ was 10. An umbilical cord haematoma $9 \mathrm{~cm}$ in length and $3 \mathrm{~cm}$ in thickness extending from the skin junction into the umbilical cord was found (Figure 3).

\section{Accepted in revised form 1 March 1985}

*Correspondence and present address: S. Ballas, M.D., Department of Obstetrics and Gynecology. Shaare Zedek Medical Center, Jerusalem 91 - 100 Israel

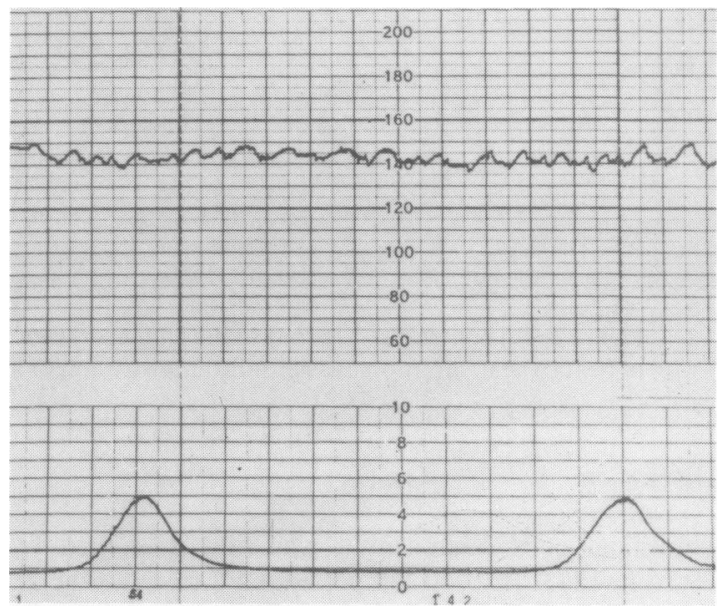

Figure 1 The baseline fetal heart rate normally exhibits varying degrees of oscillation reflective of beat to beat rate changes, which give it varying degrees of irregularity when printed out on the graphs. A normal amount of variability ( $7-14$ beats/minute) is physiological. The tracings in the upper parts of the Figures 1, 2a and 2b represent the fetal heart rate, while lines in the lower parts of these diagrams represent the uterine contractions. The vertical axis of the upper part of each diagram represents fetal heart rate in beats/min, and that of the lower part intra-uterine pressure in $\mathrm{mm} \mathrm{Hg:10.} \mathrm{The} \mathrm{horizontal} \mathrm{axis}$ in both parts of each diagram is time, each block represents 1 minute.

Microscopic examination of the cord revealed 3 blood vessels surrounded by massive haemorrhage. The point of rupture was not identified. The infant exhibited no further complications and was discharged in good condition. 


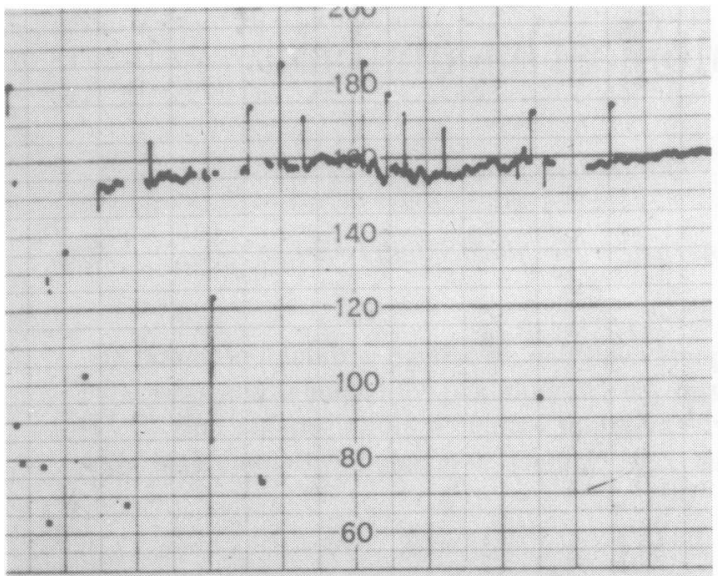

a

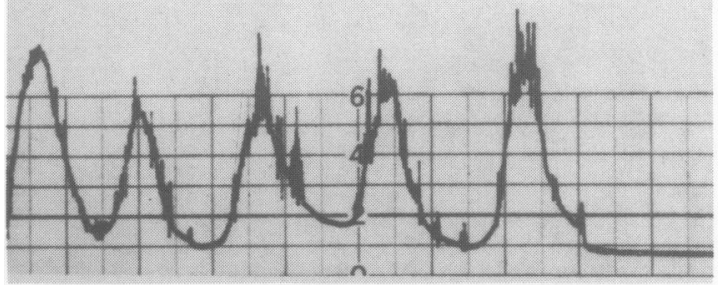

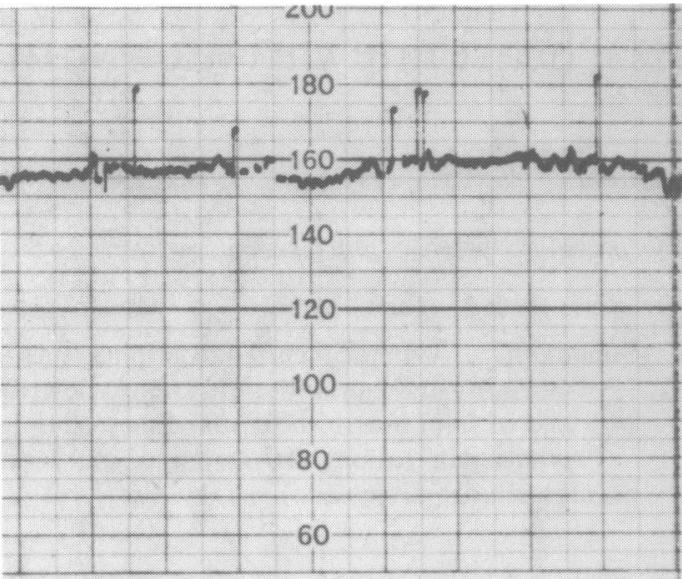

6

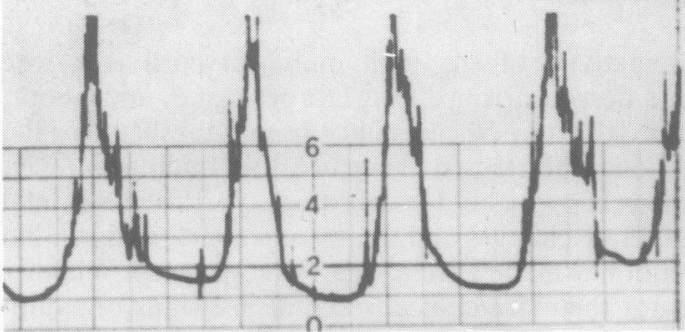

Figure 2 In contrast to Figure 1 in the fetal heart rate tracing of the patient there is no discernable oscillation-loss of beat to beat variation; (a) before administration of analgesia, (b) after administration of analgesia.

\section{Discussion}

Umbilical cord haematoma was first described by Hyrtl in 1871. In 1940 Dippel added 8 cases to the 28 reported instances up till then. Seventeen of the 36 total reported infants $(47 \%)$ were stillborn, 7 of whom expired before the onset of labour and 10 intrapartum. Only 3 infants were postmature. In 28 cases the haemorrhage arose from an identifiable rupture of the umbilical vein, in 3 from rupture of an artery and in one instance from capillaries crossing the junction between umbilical skin and adjacent cord. In 4 cases no data are available.

Breen et al. (1958) described a case in which haematoma was palpated before delivery alongside the fetal head. No fetal distress was recorded and the haematoma was due to rupture of the umbilical vein. Irani in 1964 described 2 stillbirths with umbilical cord haematoma associated with umbilical vein rupture.

In our case, the fetal heart rate tracing showed a clear decline of beat to beat variation and is the first report of umbilical haematoma with associated fetal monitoring.

The umbilical cord haematoma is most often due to rupture of either the umbilical vein, or more infrequen- tly, an umbilical artery. The following reasons have been advanced as probable factors in its development by Mary (1973), who described 3 cases.

(1) Since the majority of the reported haematomata occurred near the umbilicus, it is postulated that traction on either a short or relatively short cord, due to looping around the fetal parts, may be responsible for this location of the haematoma during descent of labour and delivery.

(2) Mechanical trauma of cord between fetal and maternal tissues may weaken the vessel wall when placental blood is forced into the umbilical vein during uterine contractions. Increasing pressure may then cause rupture of the weakened vessel wall.

(3) Either congenital weakness of a vessel wall or varix may be present.

(4) Torsion of the cord may play a part.

(5) Syphilis may represent a rare factor in its development.

The decrease of Wharton's jelly which occurs in postmaturity is postulated to predispose the cord to vessel torsion. The vessels might also be exposed to trauma, resulting in rupture of the thin walled umbilical vein.

The only antepartum clinical evidence of an 


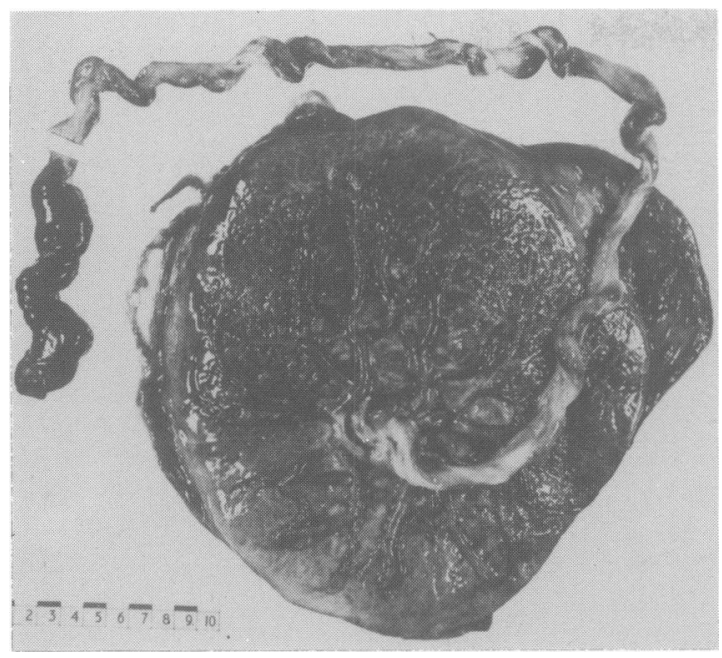

Figure 3 The placenta and the whole umbilical cord with the haematoma.

\section{References}

BREEN, J.L., RIVA, H.L. \& HATCH, R.P. (1958). Haematoma of the umbilical cord. American Journal of Obstetrics and Gynecology, 76, 1288.

DIPPEL, A.L. (1940). Haematoma of the umbilical cord. Surgery, Gynecology and Obstetrics, 70, 51.

HYRTL, J. (1871). Cited by Roberts-Thomson, M.E. (1973). umbilical cord haematoma may be fetal distress or, in severe cases, intrauterine fetal death. Fetal death or hypoxia may result from complete or partial compression of one or more of the umbilical vessels by the increasing pressure of blood in Wharton's jelly. Since no mention is made in the literature of fetal heart rate patterns, the question of pathological antepartum fetal heart rate changes in the current era of monitoring seems worthy of attention.

A clear diminution of beat to beat variation was noted during labour in the present case.

This finding, which alerts the clinician toward the diagnosis of fetal hypoxia, might be thought of as secondary to chronic prolonged (partial) umbilical cord compression. Variable decelerations, which are suggestive of the latter situation, were surprisingly absent in our case. Perhaps this contradiction points to a more chronic condition in which vessel spasm leads to fetal hypoxia. If this is true, the presence of diminished beat to beat variation, rather than variable decelerations, would become of greater diagnostic importance. Further case reports, including monitoring and $\mathrm{pH}$ data, would help to clarify this interesting issue.

The hazards of umbilical cord haematoma. Medical Journal of Australia, 1, 648.

IRANI, P.K. (1964). Haematoma of the umbilical cord. British Medical Journal, 2, 1436.

MARY, E. (1973). The hazards of umbilical cord haematoma. Australian Medical Journal, 1, 648. 